\title{
Therapeutic strategy for tracheal chondrosarcoma: report of two cases
}

\author{
Angelo Carretta $^{1}$, Paola Ciriaco ${ }^{1}$, Alessandro Bandiera ${ }^{1}$, Piergiorgio Muriana ${ }^{1}$, Gianluigi Arrigoni², \\ Giampiero Negri ${ }^{1}$ \\ ${ }^{1}$ Departments of Thoracic Surgery and ${ }^{2}$ Pathology, San Raffaele Hospital, Vita Salute San Raffaele University, Milan, \\ Italy
}

\begin{abstract}
Primary chondrosarcoma of the trachea is an extremely rare tumor. We report two cases of tracheal chondrosarcoma describing the role of surgical and conservative treatment. Endoscopic treatment with rigid bronchoscopy was performed in both patients to restore airway patency and obtain histological specimens for diagnosis. One of the patients subsequently underwent successful tracheal resection and reconstruction. The other patient, who had a contraindication to surgical treatment due to associated diseases underwent iterative endoscopic LASER treatment and is alive three years after the first diagnosis. Surgical treatment remains the treatment of choice of tracheal chondrosarcoma. When surgery is contraindicated endoscopic treatment may allow relatively longterm survival due to the slow growth of these tumors.
\end{abstract}

Correspondence: Angelo Carretta, Department of Thoracic Surgery, San Raffaele Hospital, Vita Salute San Raffaele University, Via Olgettina 60, 20132 Milan, Italy.

Tel. +39.02.26437139 - Fax +39.02.26437147.

E-mai:l angelo.carretta@hsr.it

Key words: Chondrosarcoma; trachea; surgery; bronchoscopy

Contributions: AC, concept, data acquisition, manuscript drafting; PC, concept, manuscript revision; $\mathrm{AB}$, data acquisition, literature review; PM, manuscript drafting, data collection; GA, data collection; GN, manuscript review for substantial intellectual content. All the authors have read and approved the final version of the manuscript and agreed to be accountable for all aspects of the work.

Conflict of interest: The authors declare that they have no competing interests, and all authors confirm accuracy.

Informed consent: The manuscript does not contain any individual person's data in any form.

Funding: The study was not supported by any specific funding.

Received for publication: 30 December 2019.

Accepted for publication: 25 February 2020.

${ }^{\circ}$ Copyright: the Author(s), 2020

Licensee PAGEPress, Italy

Monaldi Archives for Chest Disease 2020; 90:1223

doi: 10.4081/monaldi.2020.1223

This article is distributed under the terms of the Creative Commons Attribution Noncommercial License (by-nc 4.0) which permits any noncommercial use, distribution, and reproduction in any medium, provided the original author(s) and source are credited.

\section{Introduction}

Chondrosarcomas of the trachea are extremely rare tumors, with only 24 cases reported in the English literature to date [1-4]. Due to the low incidence of these tumors the results of different treatment options still have to be completely assessed. We present two cases of tracheal chondrosarcoma analyzing the role of endoscopic and surgical treatment.

\section{Case \#1}

A 52-years-old male patient was referred to our Department due to the recent onset of shortness of breath on exertion and hemoptysis. A CT scan showed a $30 \mathrm{~mm}$ solid vascularized lesion at the thoracic inlet infiltrating the left anterolateral side of the trachea, with intra- and extraluminal growth (Figure 1). Compression of the esophagus was also observed, without evidence of infiltration. An 18-FDG PET scan showed low tracer uptake by the lesion (Figure 2). A flexible bronchoscopy showed a vascularized lesion infiltrating the first tracheal rings with a reduction of the tracheal lumen by 30\% (Figure 3). Rigid bronchoscopy with Laser-assisted desobstruction was performed to restore airway patency and obtain specimens for pathology analysis. Histological examination showed a

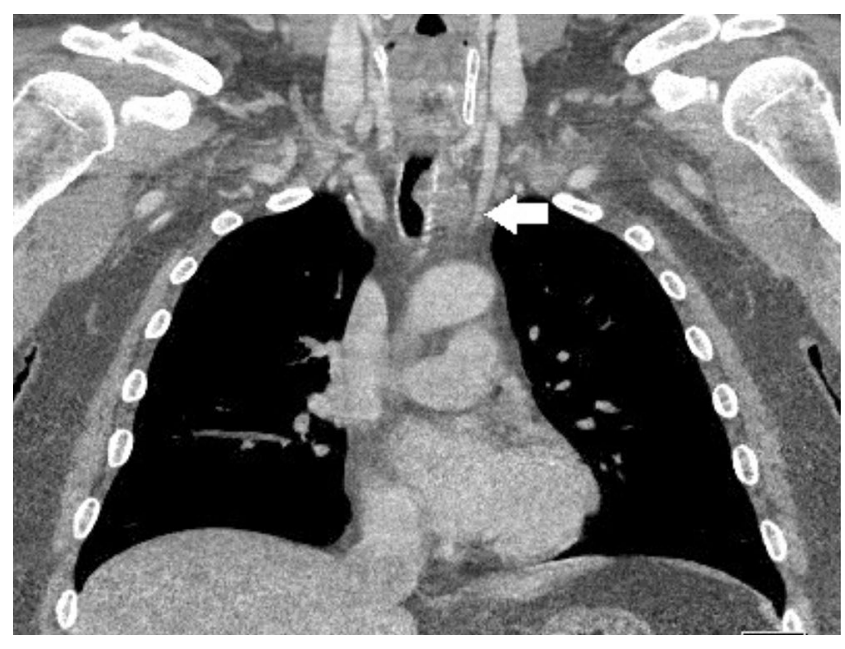

Figure 1. CT scan (coronal reconstruction) showing neoplastic lesion of the proximal trachea with intra- and extra-luminal growth (arrow). 
moderately hypercellular cartilaginous tumor with large nucleolated cells and giant nuclei, sometimes binucleated, leading to a diagnosis of moderately differentiated chondrosarcoma. Surgical treatment through cervicotomy and upper partial sternotomy was performed with a resection of a $3.5 \mathrm{~cm}$ tracheal segment (Figure 4). Intraoperative frozen section showed microscopically negative surgical resection margins, and an end-to-end tracheal reconstruction with reabsorbable interrupted suture was performed. A pedunculated cervical strap muscle was interposed between the anastomosis and the innominate artery to prevent tracheo-innominate fistula.

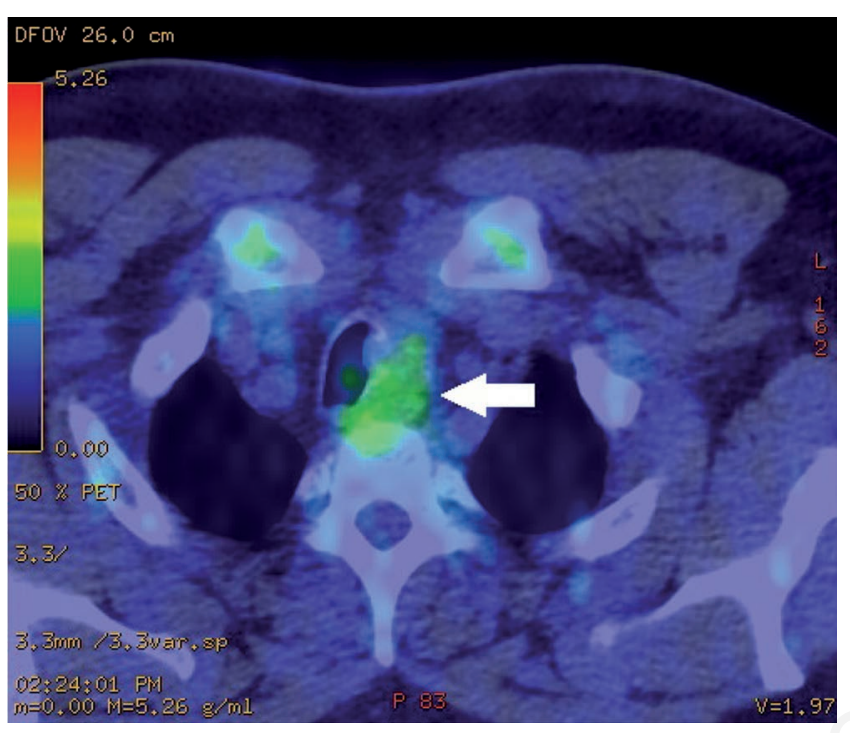

Figure 2. 18-FDG PET scan showing low tracer uptake by the tumor (arrow).

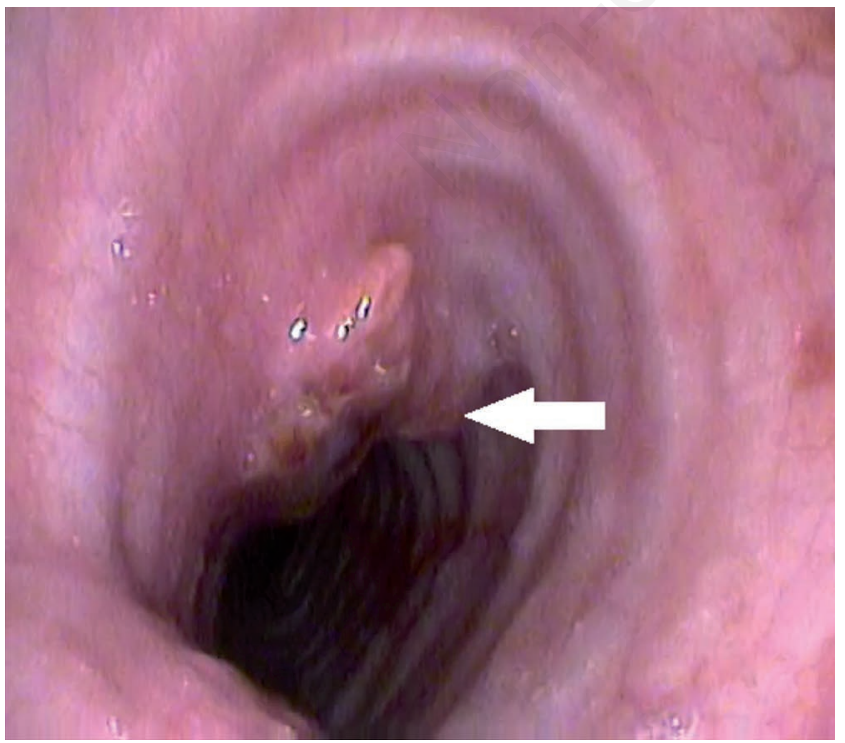

Figure 3. Preoperative flexible bronchoscopy showing neoplastic infiltration of the proximal trachea with intra-luminal extension (arrow).
Pathological examination confirmed the diagnosis of moderately differentiated (G2) tracheal chondrosarcoma with free resection margins (Figure 5). No adjuvant treatment was performed. The postoperative period was uneventful and endoscopic control at six months showed a healed and patent anastomosis, without signs of tumor recurrence (Figure 6). The patient is alive eight months after surgical treatment and further follow-up with CT scan and bronchoscopy every six months has been scheduled.

\section{Case \#2}

A 78-years old male patient was referred to our Department from the Intensive Care Unit of another hospital after failure to wean from mechanical ventilation following surgical treatment of a subdural hematoma. The patient had a previous history of pulmonary embolism, renal failure, valvular and ischemic cardiac disease. Eight years before he had undergone endoscopic resection of a tracheal chondroma. A flexible bronchoscopy performed in the ICU showed a $50 \%$ stenosis of the middle trachea due to a neoplastic lesion infiltrating the left tracheal wall of the airway, with a total length of $4 \mathrm{~cm}$. The patient underwent rigid bronchoscopy with LASER-assisted desobstruction. Rigid bronchoscopy was performed with EFER-Dumon rigid bronchoscope (EFER Endoscopy; La Ciotat, France). Endoscopic treatment by tumor coring out with the tip of the bronchoscope and diode LASER ablation (Dornier MedTech, Weßling, Germany) was performed restoring adequate airway diameter and allowing weaning from respiratory support. Histological examination of the resected tumor led to a diagnosis of G2 chondrosarcoma (Figure 7). Surgical treatment was contraindicated by the associated diseases, as also were radiotherapy and chemotherapy. One year later the patient underwent again LASER desobstruction with the same technique previously described due to intraluminal tumor recurrence significantly reducing airway diameter. Since adequate airway diameter was restored and no signs of

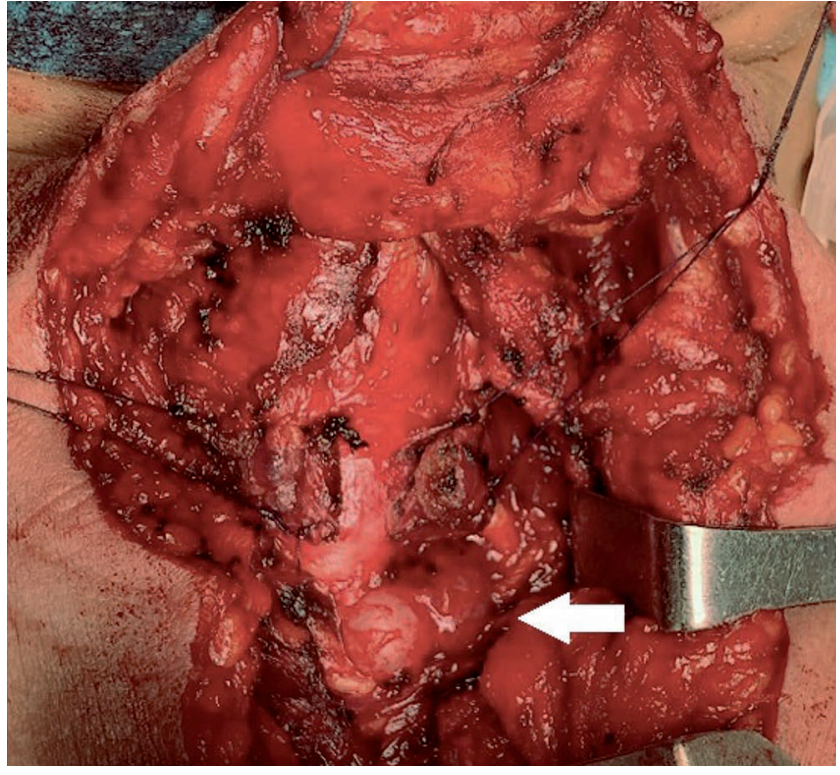

Figure 4. Intraoperative image showing extraluminal tumor growth (arrow). 
airway collapse were observed, a stent was not positioned in any of the procedures. Two years later the patient is still alive with a slow growing endotracheal tumor.

\section{Discussion}

Chondrosarcomas of the trachea are extremely rare tumors [14]. In fact, the most common sites of chondrosarcomas in the head and neck region are the larynx and maxillary region [5]. These

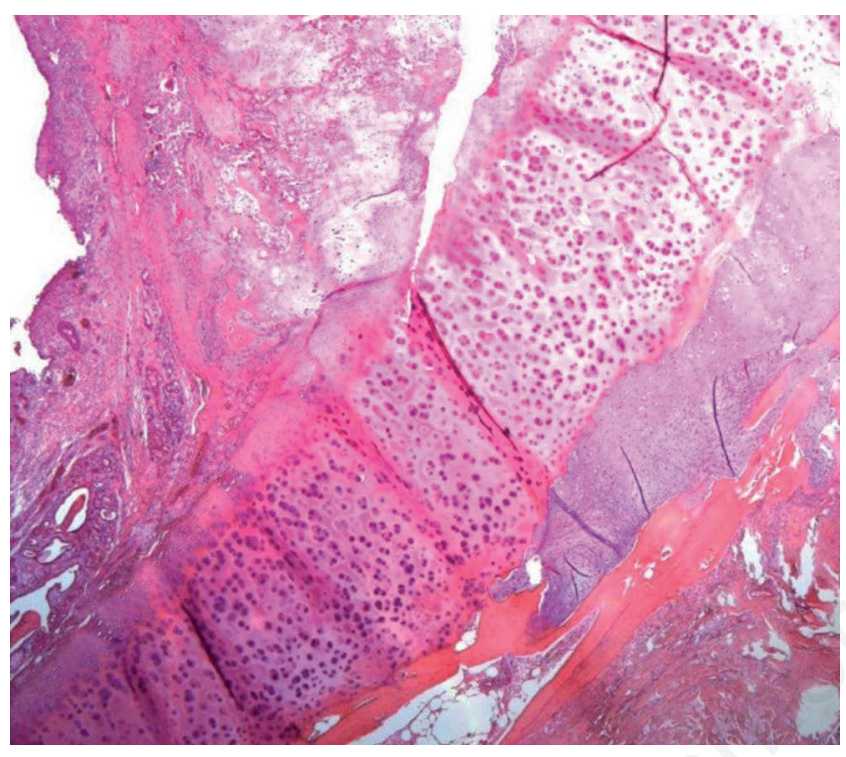

Figure 5. Histological examination showing a moderately differentiated chondrosarcoma infiltrating the tracheal cartilage and the underlying soft tissues. Hematoxylin-Eosin stain x 25 .

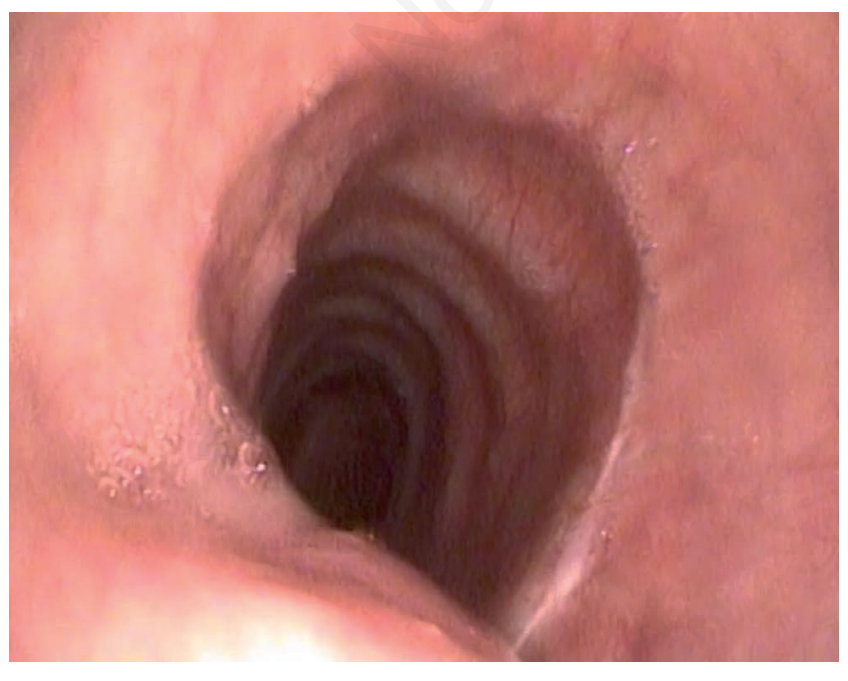

Figure 6. Videobronchoscopy six months after surgery showing a healed patent tracheal anastomosis without signs of recurrence. tumors occur more frequently in elderly male patients [6]. The two patients observed were also male, but only one of them was over 65 years of age, showing that these rare tumors may also arise in younger patients. These tumors have also been described in patients with Maffucci syndrome, a congenital disease characterized by systemic enchondromatosis and hemangiomatosis [7]. Chondrosarcomas of the head and neck usually have a slow growth, and nodal and distant metastases have been rarely reported [6]. Development of these tumors from benign chondromas has also been, although rarely, reported $[1,8]$. The history of one of the patients we observed, who had undergone endoscopic removal of a tracheal chondroma eight years before confirms this possibility. Follow-up of patients with tracheal chondromas seems therefore advisable. The most frequent symptoms associated with chondrosarcomas of the trachea are cough, dyspnea and less frequently hemoptysis [6]. Due to the slow growth of these tumors the patients are usually asymptomatic until the reduction of the airway lumen is severe [2]. Radiological diagnosis is usually performed with CT scan, which may show an intra- and extra-lumial growth. Calcifications are also frequently reported [6]. Low-grade tumors usually compress surrounding structures without infiltration. The use of PET scan was analyzed in bone chondrosarcomas, and a correlation with histological grading was observed in these tumors [9]. However, in patients with tracheal chondrosarcomas the use of PET scan had not been previously reported. In one of our patients, who had a moderately differentiated tumor, 18-FDG PET scan showed low tracer uptake. The role of PET scan may therefore be limited in the evaluation of cartilaginous tumors of the airway, although this point needs further evaluation. Bronchoscopy is the gold standard diagnostic technique in patients with tracheal chondrosarcomas, both to obtain histological specimens for a differential diagnosis and evaluate tumor extension and airway diameter in order to define the therapeutic strategy. Rigid bronchoscopy with tumor debulking to restore airway patency and obtain tumor specimens is the procedure of choice, as performed in both of our patients. The use of airway stents may also be indicated for the treatment tracheal chondrosarcomas, when LASER assisted tumor debulking does not allow to restore adequate airway patency or when airway collapse is observed. Both self-expanding metallic

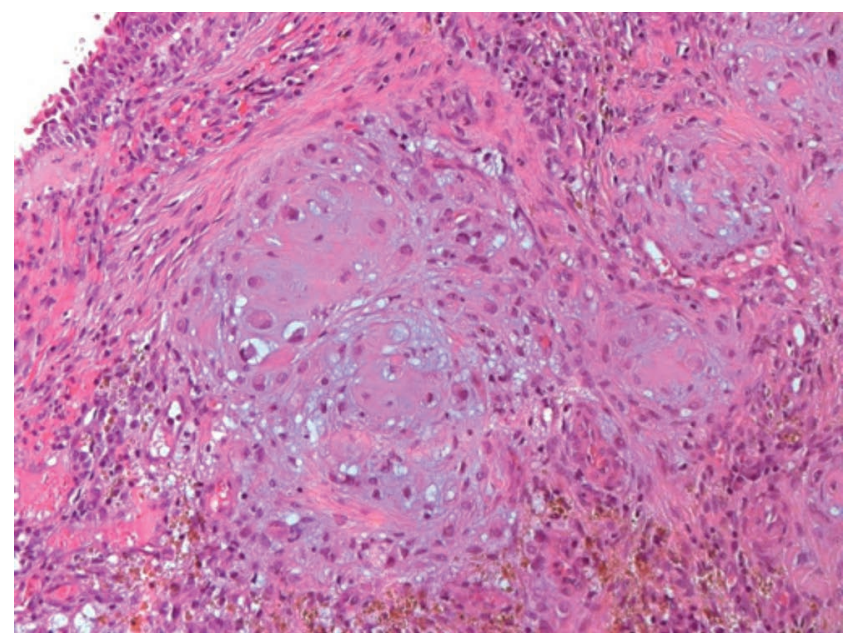

Figure 7. Moderately differentiated chondrosarcoma infiltrating the tracheal submucosa. The respiratory epithelium is seen on the left. Hematoxylin-Eosin stain x 200. 
and silicon stents may be indicated in the treatment of malignant tracheal stenosis. Points in favor of silicon stents are the fact that they can be customized and easily removed and repositioned. Conversely, metallic stents may be easier to be positioned and adapt better to airway contour, but their removal may be more demanding [10].

Different treatment options have been reported in the treatment of tracheal chondrosarcomas. Response to radiotherapy and chemotherapy is limited, although a partial response to radiotherapy has been observed $[11,12]$. Tracheal resection and reconstruction is the treatment of choice of these tumors, and a complete resolution of the disease is usually observed after complete resection $[6,13]$. Nevertheless, although surgery has to be considered the treatment of choice, relatively long-term survival may also be observed in case of low-grade tumors after endoscopic treatment despite local recurrence, as observed in one of our patients which is alive three years after the first diagnosis of chondrosarcoma.

\section{Conclusions}

Surgery remains the treatment of choice of tracheal chondrosarcomas. In patients with low grade chondrosarcomas who have a contraindication to surgical treatment iterative endoscopic desobstruction may allow relatively long-term survival due to the slow growth of these tumors.

\section{References}

1. Maia D, Elharrar X, Laroumagne S, et al. Malignant transformation of a tracheal chondroma: the second reported case and review of the literature. Rev Port Pneumol 2016;22:283-6.
2. Andolfi M, Vaccarili M, Crisci R, Puma F. Management of tracheal chondrosarcoma almost completely obstructing the airway: a case report. J Cardiothorac Surg 2016;11:101.

3. Ryabov A, Pikin O, Sokolov V, Volchenko N. Primary chondrosarcoma of the trachea with extensive extraluminal growth. Eur J Cardiothorac Surg 2017;52:601-3.

4. Khan FW, Zahid I, Moeen S, Hasan SB. Tracheal chondrosarcoma and surgical management. Asian Cardiovascular Thorac Ann 2019;27:52-4.

5. Hong P, Taylor SM, Trites JR, et al. Chondrosarcoma of the head and neck: report of 11 cases and literature review. J Otolaryngol Head Neck Surg 2009;38:279-85.

6. Kutzner EA, Park JS, Zaheer S, Inman JC. Tracheal chondrosarcoma: systematic review of tumor characteristics, diagnosis, and treatment outcomes with case report. Case Rep Oncol Med 2017;2017:45249010.

7. Wagnetz U, Patsios D, Darling G, et al. Tracheal chondrosarcoma - a rare complication in Maffucci syndrome. Br J Radiol 2009;82:e178-81.

8. Salminen US, Halttunen P, Taskinen E, Mattila S. Recurrence and malignant transformation of endotracheal chondroma. Ann Thoracic Surg 1990;49:830-2.

9. Subhawong TK, Winn A, Shemesh SS, Pretell-Mazzini J. F-18 FDG PET differentiation of benign from malignant chondroid neoplasms: a systematic review of the literature. Skeletal Radiol 2017;46:1233-9.

10. Lee P, Kupeli E, Mehta AC. Airway stents. Clin Chest Med 2010;31:141-50.

11. Obeso S, Llorente JL, Díaz-Molina JP, et al. Surgical treatment of head and neck chondrosarcomas. Acta Otorrinolaringol Esp 2010;61:262-71.

12. Coca-Pelaz A, Rodrigo JP, Triantafyllou A, et al. Chondrosarcomas of the head and neck. Eur Arch Otorhinolaryngol. 2014;271:2601-9.

13. Umezu H, Tamura M, Kobayashi S, et al. Tracheal chondrosarcoma. Gen Thorac Cardiovasc Surg 2008;56:199.202. 